\title{
Definition and Estimation of Higher-Order Gene Fixation Indices
}

\author{
Kermit Ritland \\ Department of Botany, University of Toronto, Toronto, M5S 1 A 1 Canada \\ Manuscript received March 26, 1987 \\ Revised copy accepted August 15, 1987
}

\begin{abstract}
Fixation indices summarize the associations between genes that arise from the joint effects of inbreeding and selection. In this paper, fixation indices are derived for pairs, triplets and quadruplets of genes at a single multiallelic locus. The fixation indices are obtained by dividing cumulants by constants; the cumulants describe the statistical distribution of alleles and the constants are functions of gene frequency. The use of cumulants instead of moments is necessary only for four-gene indices, when the fourth cumulant is used. A second type of four-gene index is also required, and this index is based upon the covariation of second-order cumulants. At multiallelic loci, a large number of indices is possible. If alleles are selectively neutral, the number of indices is reduced and the relationship between gene identity and gene cumulants is shown.-Two-gene indices can always be estimated from genotypic frequency data at a single polymorphic locus. Three-gene indices are also estimable except when allele frequency equals one-half. Four-gene indices are not estimable unless selection is assumed to have an equal effect upon each allele (such as under selective neutrality) and the locus contains at least three alleles of unequal frequency. For diallelic or selected loci, an alternative fourgene fixation index is proposed. This index incorporates both types of four-gene associations but cannot be related to gene identity.
\end{abstract}

$\mathrm{T}$ $\mathrm{HE}$ association of two genes at a locus can be measured either by the probability of allelic identity-by-descent (HARRIs 1964; COCKERHAM 1971) or by fixation indices based upon the covariance of allelic values (WRIGHT 1922, 1969; CockERHAM 1969; WeIR 1970). These two genes are usually considered as residing in a single diploid individual, or as having been chosen randomly from each of two diploid individuals. If one needs to consider more than two genes, computations of gene identity are relatively easily extended to an arbitrary number of genes (CANNINGS and ThOMPSON 1981). A three-gene fixation index, based upon the third moment of three genes at a diallelic locus, was derived for characterizing mating systems under nonrandom outcrossing and selection (RITLAND 1985). The extension of fixation indices to four genes, and the general multiallelic case for either three or four genes, has remained undescribed.

Measures of higher-order gene associations are useful in several ways. They can be used to find covariances of inbred relatives (GILloIs 1965, 1966). Models based upon higher-order indices of association are useful in the analyses of gene frequencies (CockERHAM 1971). In natural populations of plants, spatial associations of genotypes often occur because of genetic drift, selection and restricted gene flow. If selfing or biparental inbreeding also occurs, associations may develop among pairs of inbred individuals, and the proper characterization of these associations at one locus requires measures based upon four genes. Finally, higher-order fixation indices may be useful in selection models that incorporate such population structure.

The concept of gene identity, as conceived by MALECOT (1948), has become extremely useful for any problem that requires a measure of genetic relatedness. Gillois (1965, 1966), Cockerham (1971), JACQUARD (1974) and CANNINGS and THOMPSON (1981), and others, have used gene identity in many applications in populations and quantitative genetics. Genes are identical by descent if they are all copies of an ancestral allele. However, when selection is present, genotypic associations cannot be strictly interpreted with gene identity coefficients. In addition, estimation of gene identity requires inferences about the ancestral population to which gene identity is relative.

Alternatively, without making prior assumptions about selection and without the need to determine the relativity of the measure, we can measure the contemporary associations of alleles in terms of covariances, moments or cumulants of a distribution of allelic values. These statistical measures incorporate both the effects of inbreeding and natural selection, and are relative to the easily measured, contemporary gene frequencies.

This paper derives, in terms of gene cumulants, all two-, three-, and four-gene fixation indices for multiallelic loci. The attainable space of fixation indices 
and the estimation variance of higher-order gene fixation indices are also briefly examined. If alleles are selectively neutral, most fixation indices derived here can be related to gene identity coefficients, thus giving alternative gene cumulant definitions to higher-order gene identity coefficients.

\section{DEFINITION OF GENE FIXATION INDICES}

The statistical approach for describing associations of genotypes specifies the distribution of genotypes in terms of the cumulants of a multivariate, multinomial distribution. A sufficient number of parameters are introduced such that expected frequencies of all possible genotypes are specified. This method "saturates" or even "oversaturates" (in the case of four genes) the degrees of freedom available in the data.

In the following, we will sequentially consider the cases of two, three and four genes at a single locus. Each gene has $n$ alleles with respective frequencies $c_{1}$, $c_{2}, \ldots, c_{n}$ ( $c$ as in cumulant). Since we are considering one locus, these alleles are shared among genes and are of equal frequency among genes. At this single locus, the four genes are denoted as $a, b, c$ and $d$. For notational convenience, we always observe allele $i$ at gene $a$, allele $j$ at gene $b$, allele $k$ at gene $c$, and allele $l$ at gene $d$, even though the same allele may be observed at different genes (in which case $i=j$ for example).

Thus the assignment of alleles to genes at one locus is as follows:

$$
\begin{array}{ll}
\text { Gene } a \text { - allele } i & (i=1, \ldots, n) \\
\text { Gene } b \text { - allele } j & (j=1, \ldots, n) \\
\text { Gene } c \text { - allele } k & (k=1, \ldots, n) \\
\text { Gene } d \text { - allele } l & (l=1, \ldots, n)
\end{array}
$$

There are several specific situations to which our treatment applies. For example, if we consider only two genes, genes $a$ and $b$ can be the homologous genes of one diploid individual. For three genes, gene $c$ could additionally be the gamete allele contributed by the mate of the first individual. For four genes, genes $c$ and $d$ could additionally be the homologous genes of a second diploid individual. Other situations exist as well.

To describe the presence of alleles in genes mathematically, first consider gene $a$. For gene $a$, define a vector of Bernoulli random variables $A_{1}, A_{2}, \ldots, A_{n}$ such that if allele $i$ is present, $A_{i}=1$ and all other $A$ are zero. In other words, if allele $i$ is present, the random vector equals $(0,0, \ldots, 0,1,0, \ldots, 0)$, wherein the $i$ th term equals one.

Likewise, for gene $b$, introduce a second vector of random variables, $B_{1}, B_{2}, \ldots, B_{j}, \ldots, B_{n}$, defined such that if allele $j$ is present for gene $b, B_{j}=1$ and all other $B_{i}$ are zero. For genes $c$ and $d$, the corresponding vectors $C_{1}, \ldots, C_{k}, \ldots, C_{n}$ and $D_{1}, \ldots, D_{l}, \ldots, D_{n}$ are defined in the same way.
The matrix of random variables consisting of the four vectors is a single observation that follows a multivariate multinomial distribution. It is emphasized that the distribution function may differ between observations. This distinction is necessary only for four genes, but for consistency, is kept for lowerorder cases as well.

Two genes: First, consider genes $a$ and $b$. An observation consists of the two alleles of both genes $a$ and $b$. Observations are distributed as bivariate-multinomial with first-order cumulants denoted as $\kappa_{i}$ and $\kappa_{j}$ and second-order cumulants denoted as $\kappa_{i j}(i, j=1, \ldots, n)$. Cumulants are a set of descriptive constants of a distribution which are useful for measuring its properties, and in our circumstance, for specifying it. These cumulants are written in boldface to emphasize that their values vary among observations, and as such are random variables.

For one observation, the probability of observing allele $i$ for gene $a$ is $\mathrm{E}\left[\boldsymbol{\kappa}_{i}\right]$, the probability of observing allele $j$ for gene $b$ is $\mathbf{E}\left[\boldsymbol{\kappa}_{j}\right]$, and the probability of jointly observing alleles $i$ and $j$ is $\mathrm{E}\left[\boldsymbol{\kappa}_{i} \kappa_{j}\right]+\mathrm{E}\left[\boldsymbol{\kappa}_{i j}\right]$, where $\mathrm{E}[\mathrm{J}$ is the "expectation" operator. Cumulants about the mean of order one, two and three equal the corresponding moments about the mean. However, cumulants of order four (used in the four-gene case below) do not equal the corresponding moments of order four.

Since these cumulants may vary among observations, the population frequency $f_{i j}$ of allele $i$ for gene $a$ and allele $j$ for gene $b$ is the double expectation, taken first of single observations then taken among observations,

$$
f_{i j}=\mathrm{E}\left[\mathrm{E}\left[A_{i} B_{j}\right]\right]=\mathrm{E}\left[\kappa_{i} \kappa_{j}+\kappa_{i j}\right]=c_{i} c_{j}+c_{i j}
$$

where $c_{i}$ is the mean of $\boldsymbol{k}_{i}$ (and is the frequency of allele $i$ ), $c_{j}$ the mean of $\kappa_{j}$, and $c_{i j}$ the mean of $\kappa_{i j}$ (and is the expected covariance between alleles $i$ and $j$ ). The covariance between $\kappa_{i}$ and $\kappa_{j}$ is assumed to be zero.

To characterize the deviation of genotypic associations from Hardy-Weinberg proportions, define the following gene fixation indices (WRIGHT 1922, 1969; WEIR 1970)

$$
F_{i j}=\frac{c_{i j}}{d_{i j}} \quad(i, j=1, \ldots, n)
$$

where the denominator term $d_{i j}$ is

$$
d_{i j}=\delta_{i j} c_{i}-c_{i} c_{j}
$$

with $\delta_{i j}$ as the Kronecker operator $\left(\delta_{i j}=1\right.$ if $i=j, \delta_{j}=$ 0 otherwise). The denominator term $d_{i j}$ is the maximum value that the covariance $c_{i j}$ can take. For example, if $i=j$ then $d_{i j}=c_{i}\left(1-c_{i}\right)$, or if $i \neq j$ then $d_{i j}=$ $-c_{i} c_{j}$.

Defining the fixation indices in this way enables us 
to specify the ordered genotypic frequencies for genes $a$ and $b$ as

$$
\begin{aligned}
f_{i j} & =c_{i} c_{j}+d_{i j} F_{i j} \\
& =c_{i} c_{j}+\left(\delta_{i j} c_{i}-c_{i} c_{j}\right) F_{i j} \\
& =c_{i} c_{j}\left(1-F_{i j}\right)+\delta_{i j} c_{i} F_{i j}
\end{aligned}
$$

The last line shows how the ordered genotypic frequencies can be expressed as a fraction of genotypes in Hardy-Weinberg equilibrium and a remaining fraction of "fixed" homozygous genotypes when $i=j$. Thus the term "fixation index" for $F$ is appropriate.

If gene order is not distinguished $(i \leq j)$, then all terms in (3) with $i \neq j$ are multiplied by two (i.e., if the genotypes are heterozygous, the frequencies are doubled). More simply, if genotypes are unordered, the right side of (3) is multiplied by $\left(2-\delta_{i j}\right)$.

By taking marginal sums of $f_{i j}$ we obtain the constraints upon the expected covariances as

$$
\sum_{i=1}^{n} c_{i j}=0 \quad \text { and } \quad \sum_{j=1}^{n} c_{i j}=0 .
$$

These are standard properties of multinomial cumulants. From these constraints, we can obtain the $2 n-$ 1 constraints on the $F_{i j}$ as

$$
\sum_{i=1}^{n} c_{i} F_{i j}-\delta_{i j} F_{i j}=0 \text { and } \sum_{j=1}^{n} c_{j} F_{i j}-\delta_{i j} F_{i j}=0 .
$$

With many alleles, a large number of fixation indices can be defined. There are $n^{2}$ possible fixation indices but $(n-1)^{2}$ independent fixation indices. The $n(n-$ 1) degrees of freedom in the data (assuming equality of allele frequencies between genes $a$ and $b$ ) are saturated by $n-1$ allele frequencies plus these $(n-1)^{2}$ independent fixation indices.

Three genes: Next, consider the triplet of alleles in genes $a, b$ and $c$, this triplet being the unit of observation. The joint frequency, $f_{i j k}$, of allele $i$ for gene $a$, allele $j$ for gene $b$ and allele $k$ for gene $c$ is the expectation first taken within observations then taken among observations,

$$
\begin{aligned}
f_{i j k} & =\mathrm{E}\left[\mathrm{E}\left[A_{i} B_{j} C_{k}\right]\right] \\
& =\mathrm{E}\left[\kappa_{i} \kappa_{j} \kappa_{k}+\kappa_{i} \kappa_{j k}+\kappa_{j} \kappa_{i k}+\kappa_{k} \kappa_{i j}+\kappa_{i j k}\right], \\
& =c_{i} c_{j} c_{k}+c_{i} c_{j k}+c_{j} c_{i k}+c_{k} c_{i j}+c_{i j k},
\end{aligned}
$$

where the expected third-order cumulant between $A_{i}$, $B_{j}$ and $C_{k}$ is $c_{i j k}$, and the other $c^{\prime} s$ are as defined in (1); for example, the covariance $c_{i k}=\mathrm{E}\left[\kappa_{i k}\right]$. Covariances between first-order cumulants and second-order $\mathrm{cu}$ mulants are assumed zero.

To characterize the deviation of genotypic associations from that expected under random association of alleles, define the following three-gene fixation indices

$$
F_{i j k}=\frac{c_{i j k}}{d_{i j k}} \quad(i, j, k=1, \ldots, n) .
$$

For this three-gene index, the denominator term $d_{i j k}$ is

$$
d_{i j k}=\delta_{i j k} c_{i}-c_{i} d_{j k}-c_{j} d_{i k}-c_{k} d_{i j}-c_{i} c_{j} c_{k}
$$

where $\delta_{i j k}=1$ if $i=j=k$, or $\delta_{i j k}=0$ otherwise, and where the second-order $d$ 's are as defined in (2); for example, $d_{j k}=\delta_{j k} c_{j}-c_{j} c_{k}$. The denominator term $d_{i j k}$ is the maximum value $c_{i j k}$ can take. For example, if (a) $i=j=k$, then $d_{i j k}=c_{i}\left(1-c_{i}\right)\left(1-2 c_{i}\right)$, or if (b) $i=j$ $\neq k$, then $d_{i j k}=-c_{i} c_{k}\left(1-2 c_{i}\right)$, or finally if (c) all subscripts are unique, then $d_{i j k}=2 c_{i} c_{j} c_{k}$.

These three-gene fixation indices, together with the two-gene indices previously defined, enable us to write the three-gene frequencies as

$$
f_{i j k}=c_{i} c_{j} c_{k}+c_{i} d_{j k} F_{j k}+c_{j} d_{i k} F_{i k}+c_{k} d_{i j} F_{i j}+d_{i j k} F_{i j k},
$$

which equals, after substituting in the $\delta$ 's for the $d$ 's,

$$
\begin{aligned}
& =c_{i} c_{j} c_{k}\left(1-F_{i j}-F_{j k}-F_{i k}+2 F_{i j k}\right) \\
& \quad+\delta_{i j} c_{i} c_{k}\left(F_{i j}-F_{i j k}\right) \\
& \quad+\delta_{i k} c_{j} c_{k}\left(F_{i k}-F_{i j k}\right) \\
& \quad+\delta_{j k} c_{i} c_{j}\left(F_{j k}-F_{i j k}\right)+\delta_{i j k} c_{i} F_{i j k} .
\end{aligned}
$$

For this case of three genes, $F_{i j k}$ is the frequency that genotypes are fixed at all three genes, the $F_{i j}-F_{i j k}$ terms are frequencies that genotypes are fixed at two genes, and $\left(1-F_{i j}-F_{j k}-F_{i k}+2 F_{i j k}\right)$ is the frequency that genotypes are in random proportions.

If genotypes are unordered $(i \leq j \leq k)$, terms involving one identical pair of subscripts are multiplied by two and terms involving no identical subscripts are multiplied by six. To do this, one can multiply (7) by $6-4 \delta_{i j}-4 \delta_{i k}-4 \delta_{j k}+7 \delta_{i j k}$.

Inspection of the marginal sums of $f_{i j k}$ reveals the expected third-order cumulants have constraints

$$
\sum_{m=1}^{n} c_{i j k}=0 \text { for } m=i, j, k .
$$

From these constraints on the expected three-gene cumulants, the constraints on the three-gene fixation indices are

$$
\begin{aligned}
\sum_{k=1}^{n} 2 c_{i} c_{j} c_{k} F_{i j k}-\delta_{i k} c_{i} c_{j} F_{i j k} & -\delta_{j k} c_{i} c_{k} F_{i j k} \\
& -\delta_{i j} c_{j} c_{k} F_{i j k}+\delta_{i j k} c_{i} F_{i j k}=0
\end{aligned}
$$

and likewise for expressions involving summations over $j$ and $i$. The number of three-gene fixation indices is $n^{3}$, while the number of independent threegene indices is $(n-1)^{3}$ because of these constraints. Note that only one three-gene index is needed for a diallelic locus. The degrees of freedom in the data, $n^{3}-2(n-1)-1$, is the sum of all independent one, two and three gene parameters, $(n-1)$ $+\left[\begin{array}{l}3 \\ 2\end{array}\right](n-1)^{2}+\left[\begin{array}{l}3 \\ 3\end{array}\right](n-1)^{3}$. The description of 
genotypic frequencies is again saturated by the gene frequencies and fixation indices.

Four genes: Finally, consider the quadruplet of alleles in all four genes $a, b, c$ and $d$, which is the unit of observation. The joint frequency $f_{i j k l}$ of allele $i$ at gene $a$, allele $j$ at gene $b$, allele $k$ at gene $c$, and allele $l$ at gene $d$, is the double expectation taken within and among observations,

$$
\begin{aligned}
f_{i j k l}=\mathrm{E}[ & \left.\mathrm{E}\left[A_{i} B_{j} C_{k} D_{l}\right]\right]=\mathrm{E}\left[\kappa_{i} \kappa_{j} \kappa_{k} \kappa_{l}\right. \\
& +\kappa_{i} \kappa_{j} \kappa_{k l}+\kappa_{i} \kappa_{k} \kappa_{j l}+\kappa_{i} \kappa_{l} \kappa_{j k}+\kappa_{j} \kappa_{k} \kappa_{i l} \\
& +\kappa_{j} \kappa_{l} \kappa_{i k}+\kappa_{k} \kappa_{i} \kappa_{i j}+\kappa_{i} \kappa_{j k l}+\kappa_{j} \kappa_{i k l} \\
& +\kappa_{k} \kappa_{i j l}+\kappa_{i} \kappa_{i j k}+\kappa_{i j} \kappa_{k l}+\kappa_{i k} \kappa_{j l} \\
& \left.+\kappa_{i l} \kappa_{j k}+\kappa_{i j k l}\right] \\
=c_{i} c_{j} c_{k} c_{l}+c_{i} c_{j} c_{k l}+c_{i} c_{k} c_{j l}+c_{i} c_{l} c_{j k}+c_{j} c_{k} c_{i l} & \\
& +c_{j} c_{l} c_{i k}+c_{k} c_{l} c_{i j}+c_{i} c_{j k l}+c_{j} c_{i k l}+c_{k} c_{i j l} \\
& +c_{i} c_{i j k}+c_{i j} c_{k l}+c_{i k} c_{j l}+c_{i l} c_{j k}+\operatorname{Cov}\left[\kappa_{i j}, \kappa_{k l}\right] \\
& +\operatorname{Cov}\left[\kappa_{i k}, \kappa_{j l}\right]+\operatorname{Cov}\left[\kappa_{i l}, \kappa_{j k}\right]+c_{i j k l},
\end{aligned}
$$

where, $c_{i j k l}$ is the expected fourth-cumulant between $A_{i}, B_{j}, C_{k}$ and $D_{l}$ and Cov[...] are covariances of secondorder cumulants. The above is based upon the general formulas relating moments and cumulants given by KENDALL and STUART (1977, p. 340), with the added consideration that cumulants are variable and that pairs of second-order cumulants may covary.

To characterize the deviation of genotypic associations from that expected under random association of four alleles, the four-gene fixation indices are defined as follows:

$$
F_{i j k l}=\frac{c_{i j k l}}{d_{i j k l}} \quad(i, j, k, l=1, \ldots, n)
$$

where

$$
\begin{aligned}
d_{i j k l}= & \delta_{i j k l} c_{i}-c_{i} c_{j} c_{k} c_{l}-c_{i} c_{j} d_{k l}-c_{i} c_{k} d_{j l} \\
& -c_{i} c_{l} d_{j k}-c_{j} c_{k} d_{i l}-c_{j} c_{l} d_{i k}-c_{k} c_{l} d_{i j} \\
& -c_{i} d_{j k l}-c_{j} d_{i k l}-c_{k} d_{i j l} \\
& -c_{l} d_{i j k}-d_{i j} d_{k l} \\
& -d_{i k} d_{j l}-d_{i l} d_{j k}
\end{aligned}
$$

for $\delta_{i j k l}=1$ if $i=j=k=l$ or $\delta_{i j k l}=0$ otherwise. The lower-order $d$ 's are as defined in (2) and (6); for example, $d_{i k l}=\delta_{i k l} c_{i}-c_{i} d_{k l}-c_{k} d_{i l}-c_{l} d_{i k}-c_{i} c_{k} c_{l}$. The denominator term $d_{i j k l}$ is the maximum possible value that the expected fourth cumulant $c_{i j k l}$ can take. It is a function of allele frequencies and identities of subscripts $i, j, k$ and $l$.

A second type of four-gene measure also needs to be defined. The covariances of two-gene fixation indices are defined as:

$$
\begin{aligned}
& F_{i j \cdot k l}=\frac{\operatorname{Cov}\left[\kappa_{i j}, \kappa_{k l}\right]}{d_{i j} d_{k l}} \quad(i, j, k, l=1, \ldots, n) \\
& F_{i k . j l}=\frac{\operatorname{Cov}\left[\kappa_{i k}, \kappa_{j l}\right]}{d_{i k} d_{j l}} \quad(i, j, k, l=1, \ldots, n) \\
& F_{i l \cdot j k}=\frac{\operatorname{Cov}\left[\kappa_{i l}, \kappa_{j k}\right]}{d_{i l} d_{j k}} \quad(i, j, k, l=1, \ldots, n)
\end{aligned}
$$

where the $d$ 's are as defined in (2). The four-gene frequencies $f_{i j k l}$ thus become, in terms of fixation indices,

$$
\begin{aligned}
f_{i j k l}= & c_{i} c_{j} c_{k} c_{l}+c_{i} c_{j} d_{k l} F_{k l}+c_{i} c_{k} d_{j l} F_{j l}+c_{i} c_{l} d_{j k} F_{j k} \\
& +c_{j} c_{h} d_{i l} F_{i l}+c_{j} c_{l} d_{i k} F_{i k}+c_{k} c_{l} d_{i j} F_{i j}+c_{i} d_{j k l} F_{j k l} \\
& +c_{j} d_{i k l} F_{i k l}+c_{k} d_{i j l} F_{i j l}+c_{l} d_{i j k} F_{i j k} \\
& +d_{i j} d_{k l}\left(F_{i j} F_{k l}+F_{i j \cdot k l}\right)+d_{i k} d_{j l}\left(F_{i k} F_{j l}+F_{i k \cdot j l}\right) \\
& +d_{i l} d_{j k}\left(F_{i l} F_{j k}+F_{i l \cdot j k}\right)+d_{i j k l} F_{i j k l}
\end{aligned}
$$

which equals, after substituting in the $\delta$ 's for the $d$ 's,

$$
\begin{aligned}
f_{i j k l}= & c_{i} c_{j} c_{h} c_{l}\left[1-F_{i j}-F_{i k}-F_{i l}-F_{j k}-F_{j l}-F_{k l}\right. \\
& +F_{i j} F_{k l}+F_{i k} F_{j l}+F_{i l} F_{j k}+F_{i j \cdot k l}+F_{i k \cdot j l}+F_{i l \cdot j k} \\
& \left.+2 F_{i j k}+2 F_{i j l}+2 F_{i k l}+2 F_{j k l}-6 F_{i j k l}\right] \\
& +\delta_{i j} c_{i} c_{k} c_{l}\left[F_{i j}\left(1-F_{k l}\right)\right. \\
& \left.-F_{i j \cdot k l}-F_{i j k}-F_{i j l}+2 F_{i j k l}\right] \\
& +\delta_{i k} c_{i} c_{j} c_{l}\left[F_{i k}\left(1-F_{j l}\right)\right. \\
& \left.-F_{i k \cdot j l}-F_{i j k}-F_{i k l}+2 F_{i j k l}\right] \\
& +\delta_{i l} c_{i} c_{j} c_{k}\left[F_{i l}\left(1-F_{j k}\right)\right. \\
& \left.-F_{i l \cdot j k}-F_{i j l}-F_{i k l}+2 F_{i j k l}\right] \\
& +\delta_{j k} c_{i} c_{k} c_{l}\left[F_{j k}\left(1-F_{i l}\right)\right. \\
& \left.-F_{j k \cdot i l}-F_{i j k}-F_{j k l}+2 F_{i j k l}\right] \\
& +\delta_{j l} c_{i} c_{j} c_{k}\left[F_{j l}\left(1-F_{i k}\right)\right. \\
& \left.-F_{j l \cdot i l}-F_{i j l}-F_{j k l}+2 F_{i j k l}\right] \\
& +\delta_{k k} c_{i} c_{j} c_{k}\left[F_{k l}\left(1-F_{i j}\right)\right. \\
& \left.-F_{k l \cdot i j}-F_{i k l}-F_{j k l}+2 F_{i j k l}\right] \\
& +\delta_{i j} \delta_{k l} c_{i} c_{k}\left[F_{i j} F_{k l}+F_{i j \cdot k l}-F_{i j k l}\right] \\
& +\delta_{i k} \delta_{j l} c_{i} c_{j}\left[F_{i k} F_{j l}+F_{i k \cdot j l}-F_{i j k l}\right] \\
& +\delta_{i l} \delta_{j k} c_{i} c_{j}\left[F_{i l} F_{j k}+F_{i l \cdot j k}-F_{i j k l}\right] \\
& +\delta_{i j k} c_{j}\left[F_{i j k}-F_{i j k l}\right]+\delta_{i j l} c_{i}\left[F_{i j l}-F_{i j k l}\right] \\
& +\delta_{i k l} c_{i}\left[F_{i k l}-F_{i j k l}\right]+\delta_{j k l} c_{j}\left[F_{j k l}-F_{i j k l}\right] \\
& +\delta_{i j k l} c_{i} F_{i j k l} .
\end{aligned}
$$

This expression shows that $F_{i j k l}$ is the proportion of genotypes fixed for all four genes, the $F_{i j k}-F_{i j k l}$ terms are proportions of genotypes fixed for a triplet of genes, the $F_{i j} F_{k l}+F_{i j-k l}-F_{i j k l}$ terms are proportions fixed for two pairs of genes, the $F_{i j}\left(1-F_{k l}\right)-F_{i j \cdot k l}-$ $F_{i j k}-F_{i j l}+2 F_{i j k l}$ terms are proportions fixed for one pair of genes, and the remaining term is the proportion of genotypes in random proportions for all four alleles.

If genotypes are unordered ( $i \leq j \leq k \leq l)$, the $f_{i j k l}$ with three identical subscripts are multiplied by 2 , the $f_{i j k l}$ with two pairs of identical subscripts are multiplied by 4 , the $f_{i j k l}$ with two identical subscripts are multiplied by 6 , and the $f_{i j k l}$ with no identical subscripts are multiplied by 12. This can be accomplished by multiplying (13) by $12-6\left(\delta_{i j}+\delta_{i k}+\delta_{i l}+\delta_{j k}+\delta_{j l}+\delta_{k l}\right)+$ $2\left(\delta_{i j} \delta_{k l}+\delta_{i k} \delta_{j l}+\delta_{i l} \delta_{j k}\right)+8\left(\delta_{i j k}+\delta_{i j l}+\delta_{i k l}+\delta_{j k l}\right)-13 \delta_{i j k l}$. If genes $a$ and $b$ are unordered, and genes $c$ and $d$ unordered, but the two pairs of genes are ordered, (13) is multiplied by $\left(2-\delta_{i j}\right)\left(2-\delta_{k l}\right)$.

Marginal sums of $f_{i j k l}$ show that all expected fourth cumulants have constraints

$$
\sum_{m=1}^{n} c_{i j k l}=0 \text { for } m=i, j, k, l .
$$


From the constraints on the expected four-gene cumulants, the constraints on the four-gene fixation indices are

$$
\begin{aligned}
\sum_{l=1}^{n}- & 6 c_{i} c_{j} c_{k} c_{l} F_{i j k l}+2 \delta_{i j} c_{i} c_{k} c_{l} F_{i j k l} \\
& +\ldots-\delta_{i j} \delta_{k l} c_{i} c_{k} F_{i j k l}-\ldots \\
& -\delta_{i j k} c_{i} c_{l}\left(1-3 c_{i}\right) F_{i j k l}-\ldots+\delta_{i j k l} c_{i} F_{i j k l}=0
\end{aligned}
$$

and likewise for summations over $k, j$ and $i$. The number of four-gene fixation indices is $\left[\begin{array}{l}4 \\ 4\end{array}\right] n^{4}=n^{4}$, while the number of independent four-gene indices is $(n-1)^{4}$ because of these constraints. Note that only one four-gene index is needed for a diallelic locus. However, three covariances of indices were introduced for each independent four-gene index in (10), so the number of parameters is now $(n-1)+6(n-$ $1)^{2}+4(n-1)^{3}+4(n-1)^{4}$, which is greater than the degrees of freedom in the data, $n^{4}-3(n-1)-1$. The description of genotypic frequencies is thus oversaturated by parameters.

\section{RELATIONSHIP OF GENE CUMULANTS TO GENE IDENTITY}

This derivation allows both inbreeding and selection to structure genotypic frequencies in an arbitrary manner. Interestingly, there is a correspondence between the proportions given in the last equations of (3), (7) and (13) with those expected under identityby-descent arguments: the fixation indices which multiply the gene frequency terms $c_{i}, c_{j}, c_{k}$ and $c_{l}$ can be replaced with gene-identity coefficients to arrive at the same formulae that give joint genotypic frequencies in terms of identity coefficients, such as those given in CockerHam (1971).

If inbreeding is the only agent of gene fixation, then all $F$ with the same subscripts equal each other in expectation. For example, all $F_{i j}(i, j=1, \ldots, n)$ equal each other, all $F_{i j k}(i, j, k=1, \ldots, n)$ equal each other, all $F_{i j k l}$ equal each other, and all $F_{i j \cdot k l}$ equal each other. This drastically reduces the number of parameters needed to describe multiallelic loci. The remaining fixation indices can be related to coefficients of gene identity in the following way.

For two genes, under pure inbreeding then $F_{i j}=$ $F_{a b}(i, j=1, \ldots, n)$ and from inspection of (3), the correspondence between the two-gene fixation index and gene identity is

$$
\begin{array}{cc}
\text { Fixation index } & \text { Genes identical } \\
1-F_{a b} & \text { None } \\
F_{a b} & a=b
\end{array}
$$

where " $\equiv$ " denotes "is identical by descent to."

For three genes, again if inbreeding is the only cause of deviation from random proportions, then $F_{i j k}$ $=F_{a b c}(i, j, k=1, \ldots, n)$ and from inspection of (7), the correspondence between two- and three-gene fixation indices and two- and three-gene identities is,

$$
\begin{array}{lc}
\quad \text { Fixation indices } & \text { Genes identical } \\
1-F_{a b}-F_{a c}-F_{b c}+2 F_{a b c} & \text { None } \\
F_{a b}-F_{a b c} & a \equiv b \\
F_{a c}-F_{a b c} & a \equiv c \\
F_{b c}-F_{a b c} & \equiv \equiv c \\
F_{a b c} & a \equiv b \equiv c .
\end{array}
$$

For four genes, again if inbreeding is the only agent of gene fixation, then $F_{i j k l}=F_{a b c d}$ and $F_{i j \cdot k l}=F_{a b \cdot c d}(i$, $j, k, l, \ldots, n)$ and from inspection of (13), the correspondence between higher-order gene fixation indices and higher-order gene identities is,

$$
\begin{array}{lc}
\quad \text { Fixation indices } & \text { Genes identical } \\
1-F_{a b}-F_{a c}-F_{a d}-F_{b c} & \text { None } \\
-F_{b d}-F_{c d}+F_{a b} F_{c d} & \\
+F_{a c} F_{b d}+F_{a d} F_{b c} & \\
+F_{a b \cdot c d}+F_{a c \cdot b d} & \\
+F_{a d \cdot b c}+2 F_{a b c}+2 F_{a b d} & \\
+2 F_{a c d}+2 F_{b c d}-6 F_{a b c d} & \\
F_{a b}\left(1-F_{c d}\right)-F_{a b \cdot c d}-F_{a b c} & a \equiv b \\
-F_{a b d}+2 F_{a b c d} & \\
F_{a b} F_{c d}+F_{a b \cdot c d}-F_{a b c d} & a \equiv b \text { and } c \equiv d \\
F_{a b c}-F_{a b c d} & a \equiv b \equiv c \\
F_{a b c d} & a \equiv b \equiv c \equiv d .
\end{array}
$$

(There are six identities involving one pair, three identities involving two pairs, four identities involving triplets, plus the quadruplet and null identity, for a total of 15 terms. The reader can obtain those not given above by substitution of the lower case letters). COCKERHAM (1971) has interrelated all two-, three-, and four-gene identity coefficients between pairs, triplets and quadruplets of genes.

Condensed fixation indices: When inbreeding is the only agent of gene fixation and the four genes are those possessed by two diploid individuals, we can equate additional parameters to obtain fixation indices which are equivalent to the 9 configurations of identity (JACQUARD 1974). Let the two alleles from individual " $X$ " be genes $a$ and $b$, and the two alleles from individual " $Y$ " be genes $c$ and $d$. Since genes are exchangeable within individuals, then $F_{a c}=F_{a d}=F_{b c}$ $=F_{b d} \equiv F_{x y}, F_{a b c}=F_{a b d} \equiv F_{a b y}, F_{a c d}=F_{b c d} \equiv F_{x c d}$, and $F_{a c \cdot b d}=F_{a d \cdot c d}=F_{x \cdot y}$. By addition of the appropriate terms of (17), we obtain the following correspondence between fixation indices and gene identity: 


$$
\begin{array}{lc}
\quad \text { Fixation indices } & \text { Genes identical } \\
1-F_{a b}-F_{c d}-4 F_{x y} & \text { None } \\
+F_{a b} F_{c d}+2 F_{x y}^{2}+F_{a b \cdot c d} & \\
+2 F_{X \cdot Y}+4 F_{x c d} & a \equiv b \\
+4 F_{a b y}-6 F_{a b c d} & c \equiv d \\
F_{a b}\left(1-F_{c d}\right)-F_{a b \cdot c d} & c \equiv d F_{x c d}+2 F_{a b c d} \\
-2 F_{a b \cdot c d} & a \equiv c \text { or } a \equiv d \\
F_{c d}\left(1-F_{a b}\right)-F_{a b c d} & \text { or } b \equiv c \text { or } b \equiv d \\
-2 F_{a b y}+2 F_{a b j} & a \equiv b \text { and } c \equiv d \\
4 F_{x y}\left(1-F_{x y}\right)-4 F_{X \cdot Y} & a \equiv c \text { and } b \equiv d \\
-4 F_{x c d}-4 F_{a b y}+8 F_{a b c d} & a \equiv d \text { and } b \equiv c \\
F_{a b} F_{c d}+F_{a b \cdot c d}-F_{a b c d} & \text { or } a \equiv b \\
2 F_{x y}^{2}+2 F_{X \cdot Y}-2 F_{a b c d} & a \equiv b \equiv c \\
2 F_{a b y}-2 F_{a b c d} & \text { or } a \equiv b \equiv d \\
2 F_{x c d}-2 F_{a b c d} & a \equiv c \equiv d \\
F_{a b c d} & \text { or } b \equiv c \equiv d
\end{array}
$$

Cockerham (1971) gives a table of joint genotypic frequencies in terms of probabilities of these identity patterns.

Fully condensed fixation indices: Again for inbreeding alone, and if now the four alleles are now sampled from four different individuals in a population or if the four alleles are those of an autotetraploid (all genes are exchangeable), the correspondence between fixation indices and gene identity becomes:

$$
\begin{array}{cc}
\text { Fixation } & \text { Genes } \\
\text { indices } & \text { identical } \\
1-6 F_{2}+3 F_{2}^{2}+F_{2 \cdot 2} & \text { None } \\
+8 F_{3}-6 F_{4} & \\
6 F_{2}\left(1-F_{2}\right)-6 F_{2 \cdot 2} & \text { One pair } \\
-12 F_{3}+12 F_{4} & \\
3 F_{2}^{2}+3 F_{2 \cdot 2}-3 F_{4} & \text { Two pairs } \\
4 F_{3}-4 F_{4} & \text { Three } \\
F_{4} & \text { Four, }
\end{array}
$$

where $F_{i}$ is the fixation index of order $i$ and $F_{2 \cdot 2}$ is the covariance of second-order fixation indices.

\section{ESTIMATION OF FIXATION INDICES}

Bounds of individual indices: The two-gene fixation index ranges from -1 to +1 at a diallelic locus (WRIGHT 1969). However, when $i=j$ at a multiallelic locus, $F_{i j}$ may range down to $-\left(1-c_{i}\right) / c_{i}$ (when $c_{i}=c_{j}$ and $\mathrm{E}\left[A_{i} B_{j}\right]=c_{i}$ ). If identity by descent is the only cause of fixation, $F$ always ranges from 0 to 1 .

The three-gene fixation index $F_{i j k}$ also ranges from -1 to +1 for a diallelic locus, but at multiallelic loci when all three subscripts are unique, $F_{i j k}$ can be as low
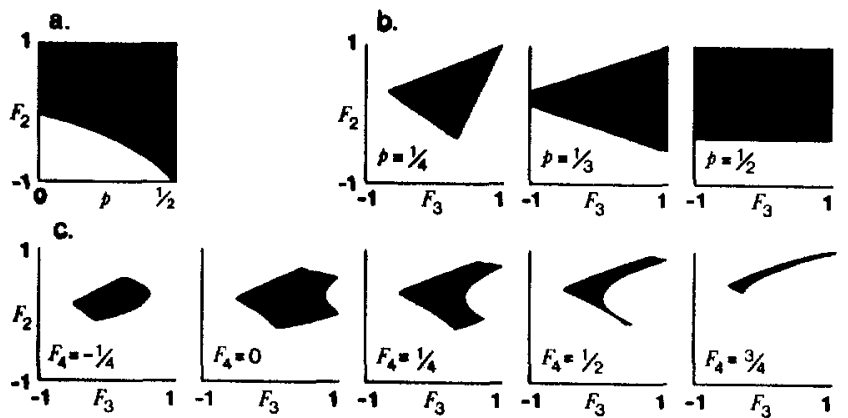

FIGURE 1.-Some examples of the space of fixation indices at a diallelic locus, as indicated by the shaded areas. (a) The space occupied by the two-gene index as a function of gene frequency $p$. (b) Space jointly occupied by the two- and three-gene indices, assuming equality of all two-gene indices, for various $p$. (c) Space of all higher-order indices, assuming equality of all indices of the same order, for $p=1 / 3$ and $F_{2 \cdot 2}=0$.

as $-\left(1-c_{i}\right) / c_{i}^{2}$ (when $c_{i}=c_{j}=c_{k}$ and $i \neq j \neq k$ ). Likewise, the four-gene fixation index $F_{i j k l}$ ranges between -1 and +1 at a diallelic locus, may be less than -1 at multiallelic loci, but always ranges from 0 to 1 if inbreeding is the only agent of gene fixation. The covariance of fixation indices $F_{i j \cdot k l}$ has a maximum determined by $F_{i j}$ and $F_{k l}$.

The space of fixation indices: THOMPSON (1976, 1980) has considered the constraints upon the "space" of genealogical relationships imposed by the mechanism of Mendelian segregation within a pedigree. When natural selection is allowed to influence genotypic frequencies in any arbitrary manner, a second class of constraints are those that merely ensure nonnegative genotypic frequencies. We can thus define the space of fixation indices (as opposed to the space of genealogical relationships) as those sets of indices which specify non-negative genotypic frequencies.

This space was examined numerically by computing genotypic frequencies throughout the space of fixation indices. The allowable space is shown in Figure 1 for some specific examples. For two genes, Figure 1a shows the allowable values of the two-gene index $F_{2}$ at a diallelic locus depends upon gene frequency. At intermediate gene frequency, this two-gene index ranges from -1 to +1 , but at more extreme frequencies, the lower limit approaches zero.

Figure $\mathrm{lb}$ shows the space of allowable two- and three-gene fully condensed indices $\left(F_{2}\right.$ and $F_{3}$ in Equation 19) for a diallelic locus. The space of fixation indices is again limited by gene frequency. In addition, their allowable values are constrained by each other. The space appears to be continuous.

Figure 1c gives slices through the space occupied by the fully condensed modes of gene identity $\left(F_{2}, F_{3}\right.$ and $F_{4}$ in Equation 19) for a diallelic locus, assuming $F_{2 \cdot 2}=0$. A small proportion of this space is occupied, and interestingly, this space appears to be discontinuous. 
Generally, the space occupied by higher-order fixation indices is quite limited. This restriction of space places limits on the confidence intervals when jointly estimating higher-order fixation indices. Second, it suggests that we should look for a set of higher-order fixation indices which occupy more of this space. Such as-yet undefined indices would presumably be functions of all lower-order cumulants.

Estimability of fixation indices: For a sample of $n$ observations, the following are method of moments estimators for cumulants, which assume that cumulants are constant:

$$
\begin{aligned}
\hat{c}_{i}= & s_{i} / n \\
\hat{c}_{i j}= & \left(n s_{i j}-s_{i} s_{j}\right) /(n(n-1)) \\
\hat{c}_{i j k}= & \left(n^{2} s_{i j k}-\sum^{3} s_{i} s_{j k}+s_{i j k}\right) /(n(n-1)(n-2)) \\
\hat{c}_{i j k l}= & \left(n^{2}(n+1) s_{j j k l}-n(n+1) \sum^{4} s_{i} s_{j k l}\right. \\
& \quad-n(n-1) \sum^{3} s_{i j} s_{k l}+2 n \sum^{6} s_{i} s_{j} s_{k l} \\
& \left.\quad-6 s_{i} s_{j} s_{k} s_{l}\right) /(n(n-1)(n-2)(n-3))
\end{aligned}
$$

where the summations are over all groupings of subscripts and where

$$
\begin{aligned}
s_{i} & =\sum^{n} A_{i} \\
s_{i j} & =\sum A_{i} B_{j} \\
s_{i j k} & =\sum_{n}^{n} A_{i} B_{j} C_{k} \\
s_{i j k l} & =\sum A_{i} B_{j} C_{k} D_{l}
\end{aligned}
$$

(KENDALL and STUART 1977, p. 329). We can obtain estimates (with some bias) of two- and three-gene fixation indices by substitution of the above estimates into (1) and (5). WEIR and CoCKERHAM (1984) discuss unbiased estimators of two-gene fixation indices.

However, if pairs of second-order cumulants covary, $\hat{c}_{i j k l}$ actually estimates $c_{i j k l}+\operatorname{Cov}\left[\kappa_{i j} \kappa_{k l}\right]+\operatorname{Cov}\left[\kappa_{i k} \kappa_{j l}\right]$ $+\operatorname{Cov}\left[\kappa_{i} \kappa_{j k}\right]$. Thus, the covariance of second-order cumulants is not separable from the fourth cumulant when cumulants are estimated with this procedure, and as a result the four-gene fixation indices, as given by (10) and (12), are not estimable with (20).

An alternative measure of four-gene associations: One solution to this lack of joint estimability of fourgene indices is to define a single fixation index that combines both types of four-gene associations. This index is defined as

$$
F_{i j k l}^{*}=\frac{\begin{array}{c}
c_{i j k l}+\operatorname{Cov}\left[\kappa_{i j}, \kappa_{k l}\right] \\
+\operatorname{Cov}\left[\kappa_{i k}, \kappa_{j l}\right]+\operatorname{Cov}\left[\kappa_{i l}, \kappa_{j k}\right]
\end{array}}{d_{i j h l}}
$$

where $d_{i j k l}$ is defined in (11). This fixation index incorporates both types of four-gene associations: (1) the tendency of all four alleles to vary together, $c_{i j k l}$, and (2) the covariance of fixed allele pairs, $\operatorname{Cov}[\ldots]$. As these fourth-order cumulants are normalized by maximum possible values, this four-gene index can range from -1 to +1 . This index is estimated by $\hat{c}_{i j k l} /$ $\hat{d}_{i j k l}$ from (20). However, $F_{j j k l}^{*}$ does not have a genetic interpretation, as it cannot be related to any pattern of gene identity.

Equivalency to a gene-identity model: A second solution to the lack of estimability of four-gene indices is to assume selection has an equal effect upon each allele (or equivalently, assume selection is absent). This introduces the constraint that all fixation indices of the same order equal each other (i.e., relations (1519) hold), and introduces enough information into the data such that four-gene parameters can be estimated. In such cases, the above method-of-moments estimators are not appropriate, and the method of maximum likelihood can be used for estimation.

When one assumes this equality of fixation indices, the equations that relate fixation indices to genotypic frequency assume the same form as equations that relate gene identity to genotypic frequency, thus suggesting one is estimating gene identity. However, estimation of gene identity also involves estimation of parameters to which gene identity is relative. The relativity of gene identity depends upon the process of evolution in the population. This process has a high variance and a significant covariance with allelic identity, and is not taken into account by the above relations.

Thus, when equality of gene fixation indices is assumed, the gene fixation model becomes equivalent to the gene identity model, but gene identity is not estimated. Rather, we estimate fixation indices which are interpretable in terms of normalized cumulants.

Variance of estimates: Five points are made here concerning the estimation variance of higher-order fixation indices. First, both the third- and fourthorder indices consist of divisors which can be zero at some gene frequency. The divisor of the three-gene index, $d_{i j k}$ (Equation 6), can be zero at a gene frequency of one-half if the subscripts $i, j$, and $k$ are not all different. The divisor of the four-gene index, $d_{i j k l}$ (Equation 11) can be zero at a gene frequency of $(3-\sqrt{3}) / 6$ if at least three subscripts are identical, or can be zero at a gene frequency of one-third if three subscripts are unique.

Figure 2a shows the divisors of the second, third and fourth cumulant plotted as a function of gene frequency at a diallelic locus. Zeros occur at a gene frequency of $(3-\sqrt{3}) / 6$ and one-half. Figure $2 b$ shows the logarithm of the standard deviation of the estimate of the corresponding cumulants. Figure 2c gives the resulting standard deviations of estimates of gene frequency. The three- and four-gene fixation indices are not estimable when divisors are zero in Figure 2a. More importantly, these indices have a high variance in the regions near these zeros.

These values are for the condensed gene fixation 

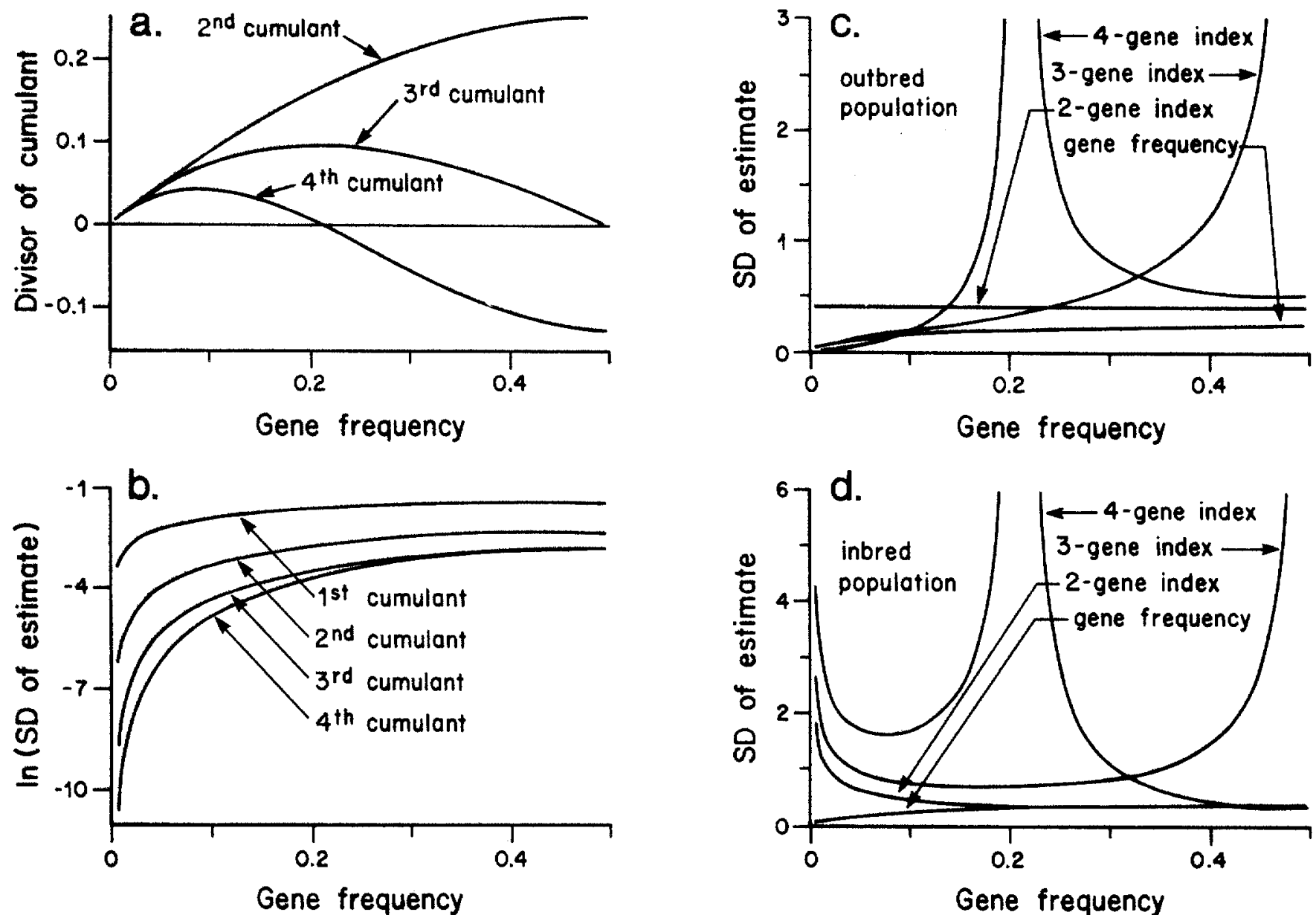

Frgure 2.- How singularities of higher-order fixation indices arise. A diallelic locus is considered and values are for single observations $(n=1)$. (a) Divisor of cumulants of a given order, as a function of gene frequency. (b) The logarithm of the standard deviation of estimates of each cumulant (assuming true values are zero). (c) Resulting standard deviations. (d) Standard deviations with inbreeding $F_{2}=0.5, F_{3}=$ $\left.0.375, F_{4}=0.25, F_{2 \cdot 2}=0\right)$.

indices (Equation 19) assuming $F_{2 \cdot 2}=0$ or equivalently for $F_{i j k l}^{*}$ (Equation 21 ), and were computed for an "outbred" population with no actual gene fixation. They were found by inversion of the information matrix, so that these are asymptotic values and functions of actual fixation indices and not sample size. All values are per observation (i.e., sample size is one). For comparison, Figure 2d gives standard deviations for a more "inbred" population $\left(F_{2}=0.5, F_{3}=0.375\right.$ and $F_{4}=0.25$ ). The same trends are apparent.

The second point is that estimates of higher-order fixation indices often have higher variance relative to the two-gene index. In Figure 2, both $c$ and $d$ show that the asymptotic variances for the three- and fourgene index are often much higher than for the twogene index. However, at most gene frequencies, these higher-order fixation indices have standard deviations only about two to three times as great as the two-gene index. In fact, at an intermediate gene frequency, the variance of the four-gene index is about equal to that of the two-gene index, although the exact relation depends on the degree of inbreeding.
The third point concerns the effect of actual inbreeding upon variance of estimates. Figure $3 a$ gives asymptotic standard deviations of estimates as a function of increasing four-gene fixation $F_{4}$ (for $F_{3}=$ $\sqrt{F_{4}}\left(1+\sqrt{F_{4}}\right) / 2, F_{2}=\sqrt{F_{4}}$ and gene frequency of one-third). Generally, variance of fixation indices decreases with increasing actual gene fixation.

The fourth point concerns effect of the number of alleles at a locus. Figure $3 \mathrm{~b}$ shows that estimation variance decreases with increasing numbers of alleles at a locus. A significant decrease of variance occurs between 3 and 4 alleles, and asymptotic variances are approached with 10 alleles at a locus. In this figure, gene fixation was assumed in the manner of Equation 18 , a triangular distribution of allele frequencies $(c$, $c / 2, c / 3, \ldots$ ) was assumed, variances were found by inverting the information matrix assuming gene frequencies were known, and the level of actual inbreeding was assumed zero. The same trend of increasing precision of estimates was found for moderate values of actual inbreeding, and for a uniform distribution of allele frequency (results not shown). 
a.
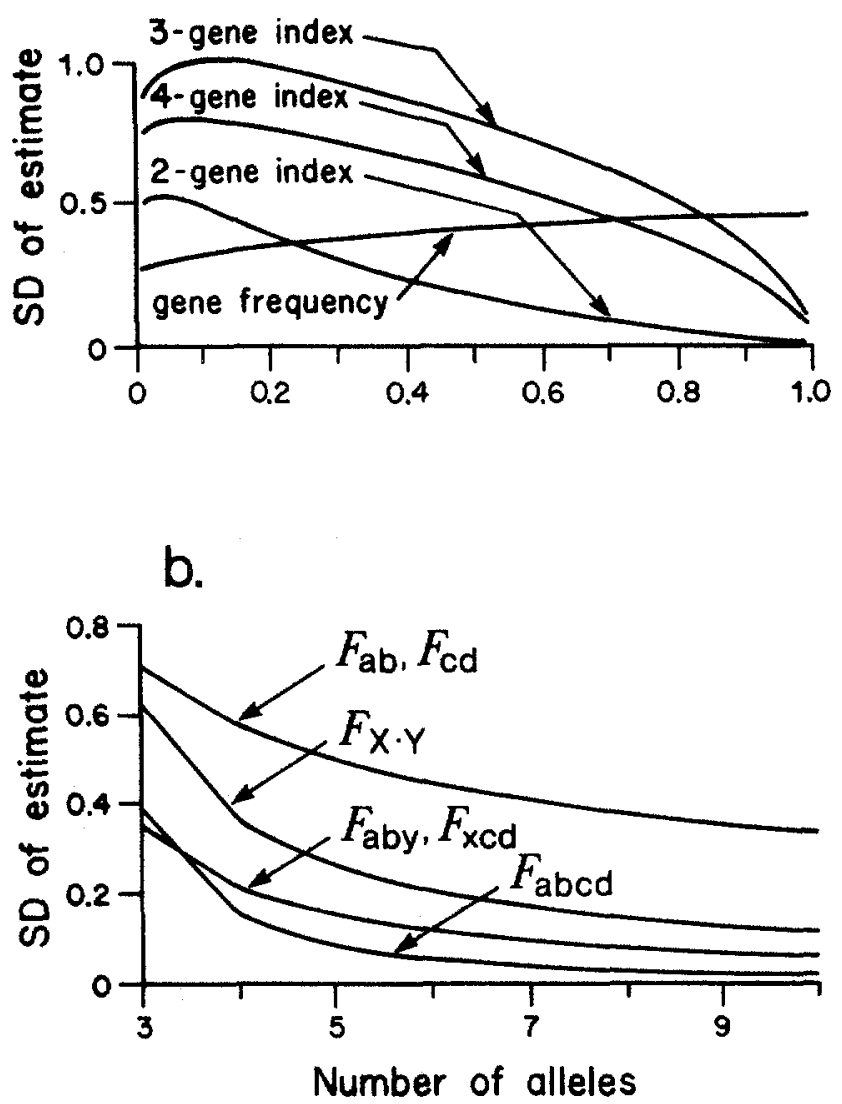

C.
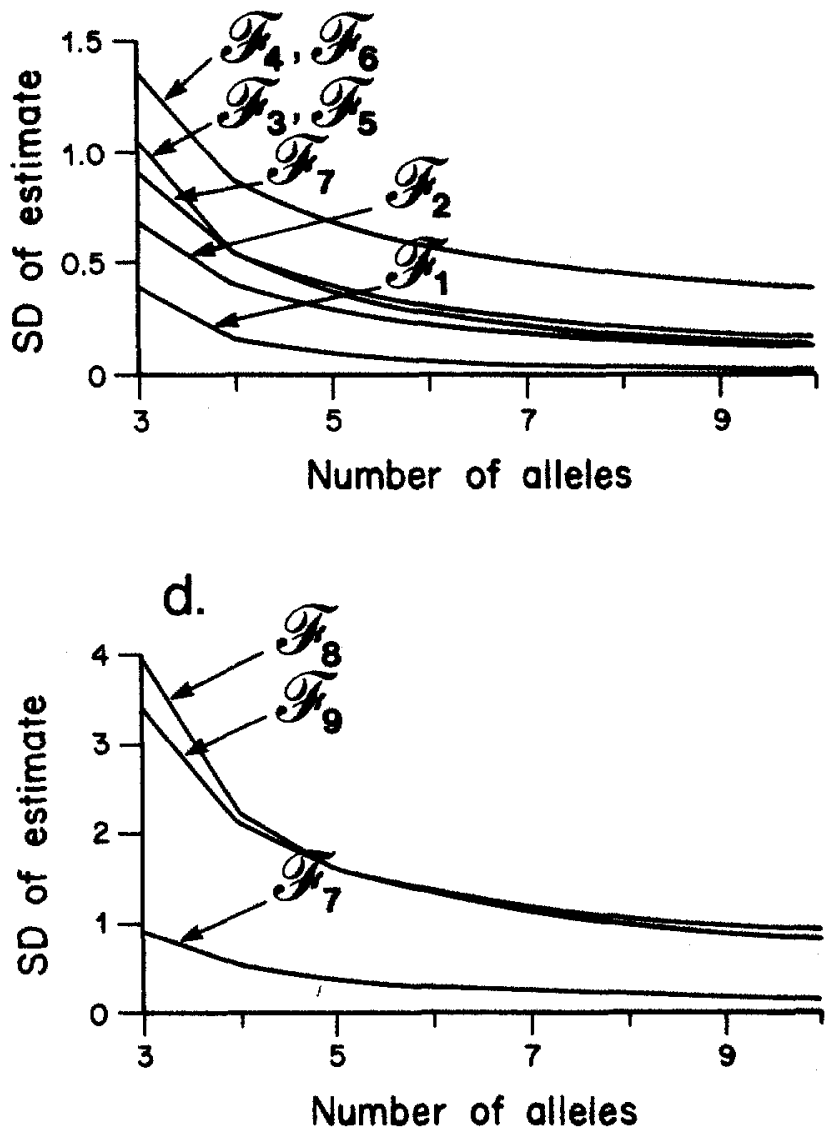

Frgure 3.-General variance properties for estimates of fixation indices. (a) Standard deviation per observation as a function of level of inbreeding $\left(F_{2}=\sqrt{F_{4}}, F_{3}=0.5 \sqrt{F_{4}}\left(1+\sqrt{F_{4}}\right), F_{2 \cdot 2}=0\right)$. (b) Standard deviations as a function of number of alleles at the locus for the fully reduced indices (Equation 19), assuming selective neutrality and a triangular distribution of allele frequency. (c and d) Standard deviations of estimates of condensed fixation indices of Equation 18 (with same conditions as b). Note: $\mathscr{F}=f(a b c d), \mathscr{F}=f(a b$ and $c d), \mathscr{F}=f(a b c$ or $a b d), \mathscr{F}_{4}=f(a b), \mathscr{F}=f(a c d$ or $b c d), \mathscr{F}=f(c d), \mathscr{F}=f(a c$ and $b d$ or $a d$ and $b c), \mathscr{F}=f(a c$ or $a d$ or $b c$ or $b d)$, fixed for same alleles are grouped, and $f$ denotes frequency.

For a diallelic locus, the information matrix was singular, showing that four-gene parameters are not estimable for a diallelic locus. This is reflected in the observation that if subscripts can take only two values in (13), the coefficients that multiply $F_{i j k l}$ and $F_{i j \cdot k l}$ in (13) are always the same. Singularity of the information matrix was also found for a triallelic locus when alleles were of equal frequency. Thus, loci must also have at least three alleles of unequal frequency to estimate four-gene indices.

The last point concerns the estimation of those gene fixations which correspond to the nine condensed identity modes. Note we cannot estimate gene identity, but rather estimate a set of fixation indices that are equivalent in the manner discussed above. Figure $3, c$ and d, shows the asymptotic standard deviations for estimates of fixation indices equivalent to the nine condensed identity modes (with same assumptions as Figure 3b). Variances of the three "outbred" indices $\mathscr{\mathscr { F } _ { 7 }}, \mathscr{F}$ and $\mathscr{F}$ were so much greater than the "inbred" indices that they were plotted separately. This suggests that our power to infer relatedness is greater for moderately inbreeding populations than for outbred populations.

\section{DISCUSSION}

The fixation index was proposed by WRIGHT (1922) as a measure of the correlation between two genes that develops as the result of the joint effects of inbreeding and selection. This paper has derived the analogous fixation indices for three and four genes. In doing so, one encounters an exponential increase of complexity from the two-gene case to the fourgene case. This complexity is somewhat misleading as the general multiallelic case was considered in this paper. For a diallelic locus with gene frequency $p=1$ $-q$, the three-gene index is $c_{3} / p q(q-p)$ where $c_{3}$ is the third cumulant of allelic values, and the two fourgene indices are $c_{4} / p q(1-6 p q)$ and and $c_{2 \cdot 2} / p^{2} q^{2}$, where $c_{4}$ is the expected fourth cumulant of allelic values and $c_{2.2}$ the covariances of second cumulants between pairs of allele values.

One might suggest that indices based upon mo- 
ments and not cumulants might be used to describe genotypic associations. However, higher-order fixation indices based upon cumulants are more appropriate because they can be directly related to gene identity-by-descent.

This equivalence between gene cumulants and gene identity has not been previously recognized. The distinction we have made between moments and cumulants is not necessary for two or three genes, but is necessary for four genes. References to gene cumulants, as opposed to moments, are lacking in related studies. WEIR and CockerHAM (1969) used fourthorder moments to study group inbreeding. YASUDA (1973) advocated the use of higher-order moments to describe mating type frequency.

In the above notation, the fourth moment $m_{4}$ equals the cumulants $c_{4}+3 c_{2.2}$. A four-gene index based upon a moment (which would equal $m_{4} / p q(1-3 p q)$ for a diallelic locus) would confound in a rather complex manner the two types of four-gene indices, given in (10) and (12).

One can conjecture that associations of more than four genes can be likewise described by appropriately normalized cumulants. However, associations between the cumulants themselves must also be considered. Equation 9 shows how the covariances between second-order cumulants enters when associations of four genes are considered. These covariances can be nuisance parameters, as they can oversaturate the data with parameters.

The estimation of four-gene parameters has been a neglected topic. Much attention has been paid to the two-gene fixation index, in large part because it is amenable to treatment by classical statistical theory such as the analysis of variance ( $c f$. WEIR and COCKERHAM 1984). Studies concentrate upon two-gene measures of relatedness (MoRTon et al. 1971; PAMILO and Crozier 1982) largely because these measures are sufficient for outbred populations. However, if we consider the four alleles as possessed by two individuals $X$ and $Y$, four-gene associations may still be present in outbred populations, as the four-gene index $F_{X \cdot Y}$ (Equation 18) can be nonzero in outbred populations.

Perhaps one reason for the lack of attention to higher-order associations is that, previously, measures have been defined only in terms of gene identity. Higher-order identity coefficients such as those given in Cockerham (1971) have always been estimable. However, estimation of gene identity requires ascertainment of the point in time to which gene identity is relative. Use of fixation indices avoids this problem because the gene cumulants are defined relative to contemporary gene frequencies.

If one assumes that selection has an equal effect upon each allele, or more likely, that selection is absent and inbreeding is the only agent of gene fixa- tion, then a locus with at least three alleles of unequal frequency provides enough information to estimate four-gene fixation indices. Generally, Figures 2 and 3 show the statistical properties of four-gene indices are reasonable. The variances of three- and four-gene fixation indices are usually larger than for two-gene indices, but barring certain allele frequencies near the singularities of Figure 2, one requires a sample only about 2-3 times larger to obtain higher-order estimates of the same precision as two-gene estimates.

Besides increasing sample size, variance can be also reduced by assaying loci with more alleles. For example, in molecular genetics, it might be desirable to screen random genomic fragments for highly polymorphic loci. However, an enormous number of alleles is by no means required, as Figure 3 shows a large reduction in variance with four or five alleles, and asymptotic variances appear to be reached by about ten alleles.

Higher-order fixation indices are difficult to relate to the classical concepts of variance and covariance, as these terms strictly apply to two variables. Twogene indices can be clearly regarded as the correlation between alleles. For four genes, the covariance of second-order indices provides some interpretation. The first type of four-gene association, $F_{i j k l}$ (Equation $10)$, might be considered as the variance of gene fixation between fixed allele pairs. The second type of four-gene association, $F_{i j \cdot k l}$ (Equation 12), is the between-pair covariance of within-pair gene fixation. The reader might gain some insight by contrasting a diallelic locus, wherein the two types of fixation are confounded, with an infinite allele locus, where the two types of fixation clearly differ.

The three-gene index seems to defy any interpretation in terms of covariances. Regardless, one point is that these higher order indices are more closely tied to covariances than to correlations, so that their range of allowable values are quite constrained compared to two-gene fixation indices (Figure 1).

At diallelic loci, the two components of four-gene association cannot be statistically separated. One solution was to define a composite four-gene fixation index $F_{i j k l}^{*}$ which includes both types of fixation (Equation 21). This four-gene index may perhaps be interpreted as simply the covariance between pairs of fixed alleles. Regression interpretations may sometimes be more appropriate. For three genes, if genes $a$ and $b$ are contained by individual $X$, and the allele contributed by the mate of $X$ is gene $c$, the effective selfing rate of this individual is $F_{a c}+F_{b c}-F_{a b c}$, which equals the coefficient of regression of the added values of $a$ and $b$ upon $c$ (RITLAND 1985).

There are many phenomena in population genetics that warrant consideration of higher-order associations of genes. These measures are particularly appropriate for plant populations, as higher-order popula- 
tion structure is predisposed to develop because of the rooted nature of plants and the tendency for nearneighbor pollination, selfing, or local seed dispersal in plant populations (BRown 1979). Functions of threegene fixation indices provide a characterization of the mating system of Mimulus guttatus (RITLAND and GANDERS 1987). For the study of correlated matings, functions of four-gene fixation indices are useful. For example, we can consider two genes as possessed by the maternal parent, and two as derived from each of two paternal parents.

Another phenomenon is variation of actual inbreeding. Among-individual variation due to variation of pedigree has been studied theoretically (WEIR, AVERY and Hill 1980; Cockerham and WeIR 1983), and its magnitude concluded to be probably small. Amongneighborhood variation of inbreeding caused by variation of effective neighborhood size is a different aspect of inbreeding variation that needs empirical determination. One approach to estimate such variation of inbreeding is to estimate four-gene fixation indices, wherein the four genes are those shared by adjacent pairs of individuals throughout a population.

Finally, higher-order fixation indices are relevant to the study of the evolutionary implications of genetic correlations between interacting individuals, or kin selection (Michod 1982). Although Michod and HAMILTON (1980) defined measures of coefficients of relatedness which are general, in the sense of being functions of higher-order inbreeding coefficients, the estimation of relatedness within social groups has apparently relied exclusively upon two-gene measures [e.g., Pamilo and Crozier (1982) and references therein], Furthermore, the assumption of weak selection in models of kin selection may be relaxed if models use fixation indices instead of gene identities. However, higher-order indices suffer from an inherent complexity that may limit their practical use.

I thank a reviewer who clarified the difference between fixation indices and gene identity coefficients, and BRUCE WEIR who provided many comments and suggested the Kronecker delta approach. This research was supported by a Natural Sciences and Engineering Research Council of Canada grant to the author.

\section{LITERATURE CITED}

Brown, A. H. D., 1979 Enzyme polymorphism in plant populations. Theor. Popul. Biol. 15: 1-42.

Cannings, C., and E. A. Thompson, 1981 Geneological and Genetic Structure. Cambridge University Press, Cambridge.
COCKERHaM, C. C., 1969 Variance of gene frequencies. Evolution 23: 72-84.

Cockerham, C. C., 1971 Higher order probability functions of identity of alleles by descent. Genetics 69: 235-246.

Cockerham, C. C., and B. S. WeIR, 1983 Variance of actual inbreeding. Theor. Popul. Biol. 23: 85-109.

GilloIs, M., 1965 Relation d'identite en génétique. I. Postulats et axiomes Mendéliens. 11. Corrélation génétique dans le cas de dominance. Ann. Inst. Henri Poincaré. Sec. B II: 1-94.

Gillors, M., 1966 Note sur la variance et al covariance génotypiques entre apparentés. Ann. Inst. Henri Poincaré. Sec. B II: 349-352.

HARRIS, D. L., 1964 Genotypic covariances between inbred relatives. Genetics 50: 1319-1348.

JACQUARD, A., 1974 The Genetic Structure of Populations. SpringerVerlag, New York.

Kendall, M., and A. Stuart, 1977 The Advanced Theory of Statistics, Vol. 1. Distribution Theory. Macmillan, New York.

Malecot, G., 1948 Les Mathematiques de l'heredite. Masson et Cie, Paris.

MichoD, R. E., 1982 The theory of kin selection. Ann. Rev. Ecol. Syst. 13: 23-55.

Michod, R. E., and W. D. Hamilton, 1980 Coefficients of relatedness in sociobiology. Nature 288: 694-697.

Morton, N. E., S. YeE, D. E. Harris and R. Lew, 1971 Bioassay of kinship. Theor. Popul. Biol. 2: 507-524.

Pamilo, P., and R. H. Crozier, 1982 Measuring genetic relatedness in natural populations: methodology. Theor. Popul. Biol. 21: 171-193.

RITLAND, K., 1985 The genetic mating structure of subdivided populations. I. Open-mating model. Theor. Popul. Biol. 27: 51-74.

Ritland, K., and F. R. GANDERs, 1987 Covariance of selfing rates with parental gene fixation indices within populations of $\mathrm{Mi}$ mulus guttatus. Evolution 41: 760-771.

Thompson, E. A., 1976 A restriction on the space of genetic relationships. Ann. Hum, Genet. 40: 201-204.

Thompson, E. A., 1980 The gene identity states of a descendent. Theor. Popul. Biol. 18: 76-93.

WeIr, B. S., 1970 Equilibria under inbreeding and selection. Genetics 65: 371-378.

WeIr, B. S., and C. C. Cockerham, 1969 Group inbreeding with two linked loci. Genetics 63: 711-742.

WerR, B. S., and C. C. Cockerham, 1984 Estimating F-statistics for the analysis of population structure. Evolution 38: 13581370.

Weir, B. S., P. J. Avery and W. G. Hill, 1980 Effect of mating structure on variation in inbreeding. Theor. Popul. Biol. 18: $396-429$

WRIGHT, S., 1922 Coefficients of inbreeding and relationship. Am. Nat. 56: 330-338.

WRIGHT, S., 1969 Evolution and the Genetics of Populations, Vol. 2. The Theory of Gene Frequencies. University of Chicago Press, Chicago.

YASUDA, N., 1973 Mating type frequency in terms of the gene frequency moments with random genetic drift. pp. 60-65. In: Genetic Structure of Populations. Edited by N. E. MORTON.

Communicating editor: B. S. WEIR 